OPEN ACCESS

Edited by:

Pinyi Lu,

Biotechnology HPC Software Applications Institute (BHSAl),

United States

Reviewed by:

Paola de Candia,

MultiMedica (IRCCS), Italy

Patrick Provost

Laval University, Canada

Xin Liu,

Stanford University, United States

${ }^{*}$ Correspondence

Martijn J. C. van Herwijnen

M.J.C.vanHerwijnen@uu.nl

tThese authors have contributed equally to this work

Specialty section:

This article was submitted to

Nutritional Immunology,

a section of the journal

Frontiers in Nutrition

Received: 31 May 2018

Accepted: 20 August 2018

Published: 18 September 2018

Citation:

Herwijnen MJCv, Driedonks TAP, Snoek BL, Kroon AMT, Kleinjan M,

Jorritsma R, Pieterse CMJ,

Hoen ENMN and Wauben MHM (2018) Abundantly Present miRNAs in Milk-Derived Extracellular Vesicles Are

Conserved Between Mammals.

Front. Nutr. 5:81.

doi: 10.3389/fnut.2018.00081

\section{Abundantly Present miRNAs in Milk-Derived Extracellular Vesicles Are Conserved Between Mammals}

\author{
Martijn J. C. van Herwijnen ${ }^{1 *}$, Tom A. P. Driedonks ${ }^{1 \dagger}$, Basten L. Snoek ${ }^{2 t}$, \\ A. M. Theresa Kroon ${ }^{1}$, Marije Kleinjan ${ }^{1}$, Ruurd Jorritsma ${ }^{3}$, Corné M. J. Pieterse ${ }^{4}$, \\ Esther N. M. Nolte-'t Hoen ${ }^{1}$ and Marca H. M. Wauben' \\ 'Department of Biochemistry and Cell Biology, Faculty of Veterinary Medicine, Utrecht University, Utrecht, Netherlands, \\ ${ }^{2}$ Theoretical Biology and Bioinformatics, Department of Biology, Science4Life, Utrecht University, Utrecht, Netherlands, \\ ${ }^{3}$ Department of Farm Animal Health, Faculty of Veterinary Medicine, Utrecht University, Utrecht, Netherlands, ${ }^{4}$ Plant-Microbe \\ Interactions, Department of Biology, Science4Life, Utrecht University, Utrecht, Netherlands
}

Mammalian milk is not only a source of nutrition for the newborn, but also contains various components that regulate further development. For instance, milk is an abundant source of microRNAs (miRNAs), which are evolutionary conserved small non-coding RNAs that are involved in post-transcriptional regulation of target mRNA. MiRNAs present in milk can occur in extracellular vesicles (EVs), which are nanosized membrane vesicles released by many cell types as a means of intercellular communication. The membrane of EVs protects enclosed miRNAs from degradation and harbors molecules that allow specific targeting to recipient cells. Although several studies have investigated the miRNA content in milk EVs from individual species, little is known about the evolutionary conserved nature of EV-associated miRNAs among different species. In this study, we profiled the miRNA content of purified EVs from human and porcine milk. These data were compared to published studies on EVs from human, cow, porcine, and panda milk to assess the overlap in the top 20 most abundant miRNAs. Interestingly, several abundant miRNAs were shared between species (e.g., let-7 family members let-7a, let-7b, let-7f, and miR-148a). Moreover, these miRNAs have been implicated in immune-related functions and regulation of cell growth and signal transduction. The conservation of these miRNA among species, not only in their sequence homology, but also in their incorporation in milk EVs of several species, suggests that they are evolutionarily selected to regulate cell function in the newborn.

Keywords: milk, miRNA, extracellular vesicles, exosomes, immune modulation

\section{INTRODUCTION}

Mother's milk provides the newborn with more than only nutrition: it contains various complex macromolecular structures that carry signaling molecules that are delivered to the newborn. One of these signaling moieties are extracellular vesicles (EVs). EVs are cell-derived lipid bilayerenclosed vesicles containing selectively incorporated proteins, lipids, and nucleic acids (mainly small RNAs) (1-3), which are in turn selectively delivered to recipient cells to modulate their functions $(4,5)$. Hence, the transfer of maternal milk EVs to the newborn allows for cross-organism communication. 
EVs are generally classified into exosomes and microvesicles, which have distinct biogenesis pathways and may differ in size and cargo $(1,2)$. Currently available methodologies do not allow discrimination between exosomes and microvesicles as the size, surface markers, and buoyant densities of these vesicle subtypes overlap (2). Therefore, we will use the generic term EVs in this study even though previously published studies attribute their findings to certain subtypes, mostly exosomes.

EVs have been identified and characterized in human milk (6$9)$, as well as in milk from other species, such as cow $(10,11)$, buffalo (12), pig (13), wallaby (14), horse (15), camel (16), rat (17), and panda (18). Additionally, extensive miRNA profiling of milk EVs has been performed in human (8-20), cow (21), pig $(13,22)$, and panda (18), and several high abundant miRNAs have been identified in each of these species. These abundantly miRNA species might be involved in the specific targeting of signaling pathways in the newborn. However, the EV isolation procedures used in these studies only enabled enrichment for milk EVs but precluded isolation of pure EVs (23). In the current study, we employed an optimized protocol that we previously developed for recovery and characterization of EVs from fresh milk $(7,9)$ in order to assess which miRNAs were most abundant in purified EVs from human and porcine milk. Additionally, we compared the identified miRNA profiles from this study with the previously reported high abundant EV-associated miRNAs from human, bovine, porcine, and panda milk. This allowed us to compare the impact of EV isolation procedures on the analysis of miRNAs in milk EVs and to examine the conserved nature of highly abundant miRNAs present in milk EVs from different species. Furthermore, we speculate on the potential impact on the newborn's development via these conserved milk EVs-associated miRNAs.

\section{METHODS}

\section{Milk EVs Isolation}

Milk EVs were isolated as previously described (7, 9). Fresh human milk from a pool of four breast-feeding mothers 3 until 9 months after delivery was obtained after informed consent of the donors and approval by the local ethics committee. Porcine milk was obtained from two sows between 2 and 3 weeks after delivery after approval by the local ethics committee. Raw milk was centrifuged twice at 3,000 $\times g$ (Beckman Coulter Allegra $\mathrm{X}-12 \mathrm{R}$, Fullerton, CA) and the milk supernatant was subjected to differential centrifugation at 5,000 $\times g$ and $10,000 \times g$ in sterilized and new SW40 tubes (Beckman Coulter). The 10,000 $\times$ $g$ supernatant was loaded onto a sucrose gradient (ranging from 2.0 to $0.4 \mathrm{M}$ sucrose) and ultracentrifuged at $192,000 \times \mathrm{g}$ (in a Beckman Coulter Optima L-90K with a SW40 rotor) for 15-18 h (k-factor 144.5). EV-containing fractions (1.12-1.18 g/ml) were harvested, pooled, and centrifuged at 100,000 $\times \mathrm{g}$ for $65 \mathrm{~min}$. After centrifugation, supernatant was removed and EV pellets were aliquoted and stored at $80^{\circ} \mathrm{C}$.

\section{EV-RNA Isolation}

Small RNA was isolated using the miRNeasy Micro kit according to the small RNA enrichment protocol provided by the manufacturer (Qiagen, Hilden, Germany). RNA yield and size profile were assessed using Agilent 2100 Bioanalyzer and Pico 6000 RNA chips (Agilent Technologies, Waldbronn, Germany) (see Table 1 for comparison between studies).

\section{Preparation of Small RNA Sequencing Libraries}

Eight nanograms of milk EV-derived small RNA was treated with DNase (Turbo DNA-free kit (Life Technologies, Carlsbad, CA), pelleted using Pellet Paint (Merck, Darmstadt, Germany), and subsequently reconstituted in $6 \mu \mathrm{l}$ milliQ water (human samples) or $12 \mu \mathrm{l}$ milliQ water (porcine samples). For the human samples, cDNA libraries were prepared using the NebNext small RNA library prep kit for Illumina (New England Biolabs, Ipswich, MA) with the following adaptations: $3^{\prime}$ adapter ligation was carried out overnight at $16^{\circ} \mathrm{C}$; Kapa HiFi Readymix $2 \times$ PCR mastermix (Kapa Biosystems, Wilmington, MA) was used for PCR amplification as follows: $2 \mathrm{~min}$ at $95^{\circ} \mathrm{C}$; 17 cycles of $20 \mathrm{~s}$ at $98^{\circ} \mathrm{C}, 30 \mathrm{~s}$ at $62^{\circ} \mathrm{C}, 15 \mathrm{~s}$ at $70^{\circ} \mathrm{C}$, and a final elongation step of $5 \mathrm{~min}$ at $70^{\circ} \mathrm{C}$. For the porcine samples, cDNA libraries were prepared as indicated by manufacturer with the Illumina TruSeq kit (Illumina Inc., San Diego, CA) with PCR amplification up to 17 cycles. cDNA was purified using AMPure XP beads (Beckman Coulter, Brea, CA) and quantified using Agilent 2100 Bioanalyzer and DNA HiSensitivity chips. Adapter dimers were removed by TBE gel purification (146-400 nt of which $126 \mathrm{nt}$ derive from adapters for human, and a size range of 150-175 bp for porcine samples).

\section{miRNA Profiling}

Data quality was checked with FastQC and reads were processed with cutadapt (v1.8) to remove low quality reads. Sequences with a minimal length of $15 \mathrm{bp}$ (human) or maximum length of $25 \mathrm{bp}$ (porcine) after adapter trimming were retained. The end-trimmed sequences were mapped to the miRNA hairpin sequences using bowtie (v1.1.1 for human and v1.2.2 for porcine) with default settings. miRNA sequences were retrieved from miRbase (v21) and for both human and porcine the combined number of reads per miRNA was used to find the top miRNAs. Supplementary File 1 lists all miRNAs identified in human and porcine milk EV, together with sequencing depth and mapping rates. Sequencing data were deposited in NCBI's Gene Expression Omnibus (GEO) under accession number GSE118409.

\section{Literature Search Previously Published Milk EVs miRNA Analysis}

To collect published data on miRNA profiling of milk EVs from different species, studies listed in PubMed up to March 2018 were selected based on the keywords "milk" and "microRNA(s)," "miRNA(s)," "extracellular vesicle(s)," "exosome(s)," or "microvesicles." Additionally, the bibliographies from the retrieved articles were searched to find additional sources. Subsequently, the experimental procedures in these papers were evaluated. Only those papers in which 
TABLE 1 | Overview of experimental details for isolation and characterization of milk EVs miRNAs in this study and previously published studies.

\begin{tabular}{|c|c|c|c|c|c|c|c|c|c|}
\hline & This study & Zhou (8) & $\begin{array}{l}\text { Simpson } \\
(20)\end{array}$ & Liao (19) & Izumi (21) & This study & Gu (13) & Chen (22) & Ma (18) \\
\hline Species & Human & Human & Human & Human & Bovine & Porcine & Porcine & Porcine & Panda \\
\hline Donors & $\begin{array}{l}\text { Pool of } 4 \\
\text { individuals }\end{array}$ & 4 Individuals & 54 Individuals & 12 Individuals & 3 Individuals & 2 Individuals & 3 Individuals & $N R$ & 3 Individuals \\
\hline $\begin{array}{l}\text { Time } \\
\text { post-partum }\end{array}$ & 3-9 months & 60 days & 3 months & 1-8 months & NR & 3-4 weeks & 0-28 days & 1-5 days & 0-30 days \\
\hline $\begin{array}{l}\text { Storage of } \\
\text { whole milk at } \\
-80^{\circ} \mathrm{C}\end{array}$ & No & Yes & Yes & Yes & Yes & No & No & Yes & No \\
\hline $\begin{array}{l}\text { EV isolation } \\
\text { procedure }\end{array}$ & $\begin{array}{l}\text { Differential } \\
\text { centrifugation; } \\
\text { density } \\
\text { gradient }\end{array}$ & $\begin{array}{l}\text { Differential } \\
\text { centrifugation; } \\
\text { Exoquick }\end{array}$ & $\begin{array}{l}\text { Differential } \\
\text { centrifugation; } \\
\text { Exoquick }\end{array}$ & Exoquick & $\begin{array}{l}\text { Differential } \\
\text { centrifugation; } \\
\text { ultra- } \\
\text { centrifugation }\end{array}$ & $\begin{array}{l}\text { Differential } \\
\text { centrifugation; } \\
\text { density } \\
\text { gradient }\end{array}$ & Exoquick & $\begin{array}{l}\text { Differential } \\
\text { centrifugation; } \\
\text { ultra- } \\
\text { centrifugation }\end{array}$ & $\begin{array}{l}\text { Differential } \\
\text { centrifugation; } \\
\text { Exoquick }\end{array}$ \\
\hline $\begin{array}{l}\text { miRNA } \\
\text { extraction kit }\end{array}$ & miRNeasy & TRIzol-LS & miRNeasy & TRlzol & miRNeasy & miRNeasy & TRIzol-LS & TRIzol & TRIzol-LS \\
\hline $\begin{array}{l}\text { Library } \\
\text { preparation } \\
\text { kit }\end{array}$ & $\begin{array}{l}\text { New England } \\
\text { Biolabs, } \\
\text { NebNext }\end{array}$ & $N R$ & $\begin{array}{l}\text { Epicentre } \\
\text { Biotechnologies, } \\
\text { ScriptMiner }\end{array}$ & $\begin{array}{l}\text { Illumina } \\
\text {, TruSeq }\end{array}$ & $\begin{array}{l}\text { Not } \\
\text { applicable }\end{array}$ & $\begin{array}{l}\text { Illumina } \\
\text { TruSeq }\end{array}$ & $N R$ & $N R$ & NR \\
\hline $\begin{array}{l}\text { miRNA } \\
\text { profiling } \\
\text { method }\end{array}$ & $\begin{array}{l}\text { HiSeq } 2000 \\
\text { (Illumina) }\end{array}$ & $\begin{array}{l}\text { Genome } \\
\text { Analyzer II } \\
\text { (Illumina) }\end{array}$ & $\begin{array}{l}\text { HiSeq } 2000 \\
\text { (Illumina) }\end{array}$ & $\begin{array}{l}\text { HiSeq } 2500 \\
\text { (Illumina) }\end{array}$ & $\begin{array}{l}\text { Microarray } \\
\text { ( } 670 \text { bovine } \\
\text { miRNA) }\end{array}$ & $\begin{array}{l}\text { HiSeq } 2000 \\
\text { (Illumina) }\end{array}$ & $\begin{array}{l}\text { Genome } \\
\text { Analyzer II } \\
\text { (Illumina) }\end{array}$ & $\begin{array}{l}\text { Solexa } \\
\text { sequencing }\end{array}$ & $\begin{array}{l}\text { Genome } \\
\text { Analyzer II } \\
\text { (Illumina) }\end{array}$ \\
\hline
\end{tabular}

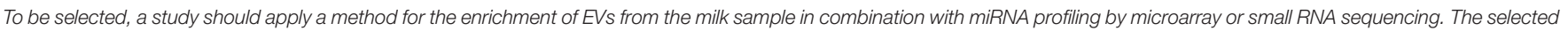

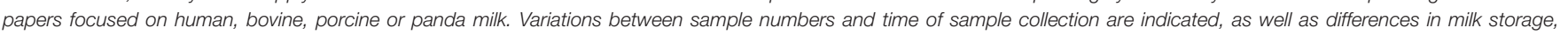
EVs/miRNA isolation procedures, and miRNA profiling methods. NR, not reported.

extensive miRNA profiling was performed either by deep sequencing or microarray on milk samples enriched (by differential centrifugation and/or precipitation) for milk $\mathrm{EVs}$ were selected for further comparison. In total seven studies were selected to compare the most abundantly present miRNAs (see Table 1 for comparison of selected studies).

\section{In silico Analysis}

Tarbase (24) was used to screen for validated targets of identified miRNAs and TargetScan (25) was used to assess conservation of these target sites. Funrich (26) was used for GO analysis on the identified targets.

\section{RESULTS}

\section{miRNA Profiling of Purified EVs From Human and Porcine Milk}

We show for the first time the miRNA profile of purified milk EVs isolated after differential centrifugation followed by density gradient separation. We identified 309 mature miRNAs in human milk EVs and 218 mature miRNAs in porcine milk EVs (Supplementary File 1), These numbers are in range with the miRNAs that were previously identified in milk EV enriched samples of human $[n=602$ (8), $n=125$ (20) and $n=610$ (19); average of $446 \pm 278$ miRNAs $]$ and porcine $[n=234$ (13) and $n=491$ (22); average of 363 \pm 182 miRNAs]. Hence, the number of identified miRNAs seems not to differ greatly between purified EVs and EVenriched samples or the RNA extraction and profiling methods used.

\section{Milk EVs From Different Species Share Abundantly Present miRNAs}

In order to identify similarities in the miRNA composition of milk EVs from different species, we compared the top 20 most abundant miRNAs detected in purified milk EVs to the reported top 20 most abundant miRNAs in the selected publications. We observed substantial overlap in the top-ranked miRNAs observed in the different studies, with 19 miRNAs being abundantly detected in at least four out of nine studies (Table 2). Interestingly, four miRNAs were identified in high abundance in all four species examined. These included miRNA let-7 family members let-7a, let-7b, and let-7f, as well as miR148a. These miRNAs were fully conserved at the sequence level in all species (Supplementary File 1). In addition to the similarities observed in miRNA content of EV, we also identified miRNAs that were abundantly present in milk EVs from all but one species. For example, miR-20a, miR-26a, and miR-141 were not present in the top 50 most abundant miRNAs in any of the three porcine studies, while these miRNAs are in the porcine genome. Additionally, let-7c was not abundantly present in any of the four human studies (see Supplementary File 1 for the full Table 2). Furthermore, we also observed differences in miRNA profiles reported in studies investigating milk EVs from the same species. Many factors may underlie these differences, including pre-analytic variables, differences in milk EVs and RNA isolation protocols, 
TABLE 2 | Overlap in top 20 most abundant miRNAs detected in milk EVs from human, cow, pig, and panda.

\begin{tabular}{|c|c|c|c|c|c|c|c|c|c|c|}
\hline $\begin{array}{l}\text { Top } 20 \\
\text { ranked }\end{array}$ & $\begin{array}{l}\text { This } \\
\text { study }\end{array}$ & $\begin{array}{l}\text { Zhou et } \\
\text { al. (8) }\end{array}$ & $\begin{array}{l}\text { Simpson et } \\
\text { al. (20) }\end{array}$ & $\begin{array}{l}\text { Liao et } \\
\text { al. (19) }\end{array}$ & $\begin{array}{l}\text { Izumi et } \\
\text { al. (21) }\end{array}$ & $\begin{array}{l}\text { This } \\
\text { study }\end{array}$ & $\begin{array}{l}\text { Gu et al. } \\
\text { (13) }\end{array}$ & $\begin{array}{l}\text { Chen et } \\
\text { al. (22) }\end{array}$ & $\begin{array}{l}\text { Ma et al. } \\
(18)\end{array}$ & $\begin{array}{l}\text { Times } \\
\text { identified } \\
\text { in top } 20\end{array}$ \\
\hline miRNA & Human & Human & Human & Human & Bovine & Porcine & Porcine & Porcine & Panda & \\
\hline let-7a-5p & 5 & 6 & 6 & 17 & 8 & 1 & 10 & 8 & 5 & 9 \\
\hline $\begin{array}{l}\text { miR-148a- } \\
3 p\end{array}$ & 2 & 1 & 1 & 2 & 14 & & 1 & $\bullet$ & 2 & 7 \\
\hline miR-30a-5p & 10 & NR & 13 & 11 & $\bullet$ & 2 & 2 & 13 & 8 & 7 \\
\hline let-7f-5p & 9 & 3 & 7 & $\bullet$ & 17 & 6 & NR & 9 & 13 & 7 \\
\hline miR-30d-5p & 1 & NR & 3 & 4 & $\bullet$ & 5 & 5 & $\bullet$ & 17 & 6 \\
\hline let-7b-5p & 8 & NR & 4 & 15 & 4 & & NR & $\bullet$ & 1 & 5 \\
\hline$m i R-21-5 p$ & 7 & NR & 10 & $\bullet$ & & 4 & 12 & $\bullet$ & 15 & 5 \\
\hline miR-22-3p & 18 & NR & 2 & 1 & & $\bullet$ & NR & 17 & 16 & 5 \\
\hline miR-320a-3p & 17 & NR & $\bullet$ & 20 & 15 & 10 & NR & 3 & $\bullet$ & 5 \\
\hline$m i R-191-5 p$ & $\bullet$ & $N R$ & $\bullet$ & 13 & & 3 & 9 & 7 & $\bullet$ & 4 \\
\hline miR-200a-3p & 3 & 9 & 5 & 16 & $\bullet$ & & NR & & $\bullet$ & 4 \\
\hline miR-181a-5p & $\bullet$ & NR & $\bullet$ & 3 & & 20 & NR & 4 & 10 & 4 \\
\hline miR-92a-3p & $\bullet$ & NR & $\bullet$ & 8 & & 13 & NR & 14 & 3 & 4 \\
\hline$m i R-182-5 p$ & $\bullet$ & 8 & & 10 & & 19 & 4 & & & 4 \\
\hline miR-141-3p & & 7 & 20 & 5 & 10 & & NR & & $\bullet$ & 4 \\
\hline let-7g-5p & 11 & NR & $\bullet$ & 14 & $\bullet$ & 9 & NR & $\bullet$ & 9 & 4 \\
\hline let-7c & & NR & & & 13 & 7 & NR & 10 & 19 & 4 \\
\hline miR-375-3p & 14 & NR & 17 & 9 & $\bullet$ & & 13 & & & 4 \\
\hline miR-26a-5p & $\bullet$ & NR & 18 & 6 & 20 & & NR & & 20 & 4 \\
\hline miR-200c-3p & 4 & NR & $\bullet$ & $\bullet$ & 9 & 8 & NR & & $\bullet$ & 3 \\
\hline
\end{tabular}

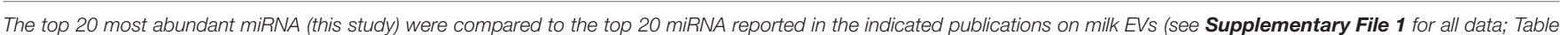

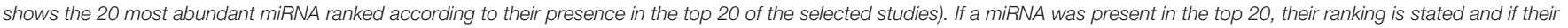

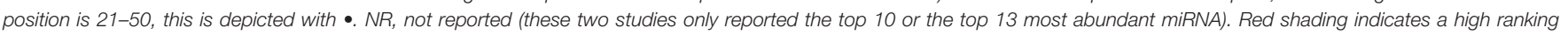

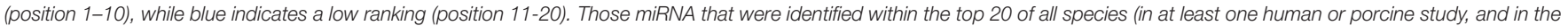
bovine and panda study) are depicted in bold.

time in lactation period, and inter-individual differences in milk composition.

\section{Conserved Milk EV-Associated miRNAs Have Immune-Related Functions}

Milk EVs have been indicated to modulate immune cells (6) and promote epithelial cell growth (27). Importantly, milk-derived miRNAs have been shown to survive harsh conditions including RNase digestion $(8,13,14,18)$, low $\mathrm{pH}(13,14,18)$, and in vitro gastro-intestinal digestion $(19,28)$. This indicates that milk EVs resist gastro-intestinal conditions and therefore presumably remain intact in the newborn gastro-intestinal tract. Even though no strong direct in vivo evidence exists, milk EVs can be taken up in vitro by intestinal epithelial cells $(19,29,30)$, vascular epithelial cells (31), and macrophages $(11,21,32)$. This suggests that EV cargo can reach the cells at the mucosa of the newborn.

In order to link the conserved milk EV miRNAs to relevant physiological processes, we identified 24 validated target (Supplementary File 1) and used GO analysis to determine that these targets are involved in "cell growth and/or maintenance," "cell communication" and "signal transduction" (Supplementary File 1). In fact, let-7a/b/f-5p and miR-148-3p have been shown to regulate signal transduction by downregulating the transcription factor NF- $\kappa \mathrm{B}$ resulting in a dampened immune response (33-35). Collectively, these data suggest that milk EVs harbor evolutionary miRNAs with immunomodulatory functions that can regulate the newborns development.

\section{DISCUSSION}

In this study, we isolated EVs from human and porcine milk and determined the presence and abundance of miRNAs. The top 20 of most abundantly present miRNAs from the purified human or porcine milk EVs were compared to previously reported miRNAs that were profiled from EV-enriched samples from human, bovine, porcine, and panda milk. Four specific miRNAs appeared to be highly abundant and conserved not only in their sequence, but also in their presence in milk of several species.

At first glance the number of isolated miRNA species does not seem to differ greatly between purified and EV-enriched milk samples and the four conserved miRNAs were identified irrespective of further EV purification and disparate RNA isolation and profiling methods. However, we also observed clear differences along the line reported earlier by van Deun et al. (36). For instance, miR-100-5p was only detected in 
high abundance in the purified human milk EVs from this study and was not present in the top 50 of other human studies. In contrast, miR-30b-5p was not abundantly present in purified human milk EV, while it was detected in the three other human studies. For porcine milk EVs there were many discrepancies between the identification of miRNAs between purified EVs compared to the other isolation methods. This could also be caused by a divergence in RNA isolation, library preparations, and sequencing method, which was different for this study compared to $\mathrm{Gu}$ et al. and Chen et al. A first step would be to standardize milk collection, milk storage, milk EV isolation, miRNA extraction, miRNA profiling, and data analysis. Finally, adequate reporting of experimental details should be a priority in the EV field to increase rigor and reproducibility (37).

In conclusion, the evolutionary conserved character of a selected set of miRNAs in milk EVs is remarkable. Their presence in EVs suggests a possible conserved role in maternal to newborn cross-organism communication. By targeting a variety of genes, including those that are involved in the regulation of the newborn's epithelial barrier and immune system these maternal milk EV-associated miRNAs might contribute to the guided further development of the newborn.

\section{ETHICS STATEMENT}

For human subjects: This study was carried out in accordance with the recommendations of Medical Research Involving Human Subjects Act (WMO), Medical Research Ethics Committee UMC Utrecht. The protocol was approved by

\section{REFERENCES}

1. van der Grein SG, Nolte-'t Hoen EN. "Small talk" in the innate immune system via RNA-containing extracellular vesicles. Front Immunol. (2014) 5:542. doi: 10.3389/fimmu.2014.00542

2. van Niel G, D'Angelo G, Raposo G. Shedding light on the cell biology of extracellular vesicles. Nat Rev Mol Cell Biol. (2018) 19:213-28. doi: $10.1038 / \mathrm{nrm} .2017 .125$

3. Guduric-Fuchs J, O’Connor A, Camp B, O’Neill CL, Medina RJ, Simpson DA. Selective extracellular vesicle-mediated export of an overlapping set of microRNAs from multiple cell types. BMC Genomics (2012) 13:357. doi: 10.1186/1471-2164-13-357

4. Mittelbrunn M, Gutierrez-Vazquez C, Villarroya-Beltri C, Gonzalez S, Sanchez-Cabo F, Gonzalez MA, et al. Unidirectional transfer of microRNAloaded exosomes from T cells to antigen-presenting cells. Nat Commun. (2011) 2:282. doi: 10.1038/ncomms1285

5. Montecalvo A, Larregina AT, Shufesky WJ, Stolz DB, Sullivan ML, Karlsson JM, et al. Mechanism of transfer of functional microRNAs between mouse dendritic cells via exosomes. Blood (2012) 119:756-66. doi: 10.1182/blood-2011-02-338004

6. Admyre C, Johansson SM, Qazi KR, Filen JJ, Lahesmaa R, Norman M, et al. Exosomes with immune modulatory features are present in human breast milk. J Immunol. (2007) 179:1969-78. doi: 10.4049/jimmunol.179. 3.1969

7. Zonneveld MI, Brisson AR, van Herwijnen MJ, Tan S, van de Lest $\mathrm{CH}$, Redegeld FA, et al. Recovery of extracellular vesicles from human breast milk is influenced by sample collection and vesicle isolation procedures. J Extracell Vesicles (2014) 3:24215. doi: 10.3402/jev.v3.24215 the Medical Research Ethics Committee UMC Utrecht. All subjects gave written informed consent in accordance with the Declaration of Helsinki. For animal subjects: This study was carried out in accordance with the recommendations of guideline 2010/63/EU, dierexperimentencommissie (DEC) Utrecht, in which no approval for milk sampling under the described conditions was required.

\section{AUTHOR CONTRIBUTIONS}

$\mathrm{MH}, \mathrm{RJ}, \mathrm{CP}, \mathrm{EH}$, and MW conceived and designed the study. $\mathrm{MH}, \mathrm{TD}, \mathrm{BS}, \mathrm{AK}$, and $\mathrm{MK}$ performed experiments and analyzed the data. $\mathrm{MH}, \mathrm{TD}, \mathrm{BS}, \mathrm{AK}, \mathrm{EH}$, and $\mathrm{MW}$ wrote and revised the manuscript. All authors contributed to editing the paper.

\section{FUNDING}

This work was supported by a Future Food Utrecht seed fund (to MW, CP, RJ), Netherlands Organization for Scientific Research (NWO Enabling Technologies project nr 435002022) (to MW, $\mathrm{EH}$ ), the European Research Council under the 441 European Union"s Seventh Framework Programme (FP/2007-2013)/ERC Grant Agreement number 442337581 (to EH), and an ERC Advanced Grant 269072 (to CP).

\section{SUPPLEMENTARY MATERIAL}

The Supplementary Material for this article can be found online at: https://www.frontiersin.org/articles/10.3389/fnut.2018. 00081/full\#supplementary-material
8. Zhou Q, Li M, Wang X, Li Q, Wang T, Zhu Q, et al. Immune-related microRNAs are abundant in breast milk exosomes. Int J Biol Sci. (2012) 8:118-23. doi: 10.7150/ijbs.8.118

9. van Herwijnen MJ, Zonneveld MI, Goerdayal S, Nolte-’t Hoen EN, Garssen J, Stahl B, et al. Comprehensive proteomic analysis of human milkderived extracellular vesicles unveils a novel functional proteome distinct from other milk components. Mol Cell Proteomics (2016) 15:3412-23. doi: 10.1074/mcp.M116.060426

10. Blans K, Hansen MS, Sorensen LV, Hvam ML, Howard KA, Moller A, et al. Pellet-free isolation of human and bovine milk extracellular vesicles by size-exclusion chromatography. J Extracell Vesicles (2017) 6:1294340. doi: 10.1080/20013078.2017.1294340

11. Pieters BC, Arntz OJ, Bennink MB, Broeren MG, van Caam AP, Koenders MI, et al. Commercial cow milk contains physically stable extracellular vesicles expressing immunoregulatory TGF-beta. PLoS ONE (2015) 10:e0121123. doi: 10.1371/journal.pone.0121123

12. Baddela VS, Nayan V, Rani P, Onteru SK, Singh D. Physicochemical biomolecular insights into buffalo milk-derived nanovesicles. Appl Biochem Biotechnol. (2016) 178:544-57. doi: 10.1007/s12010-015-1893-7

13. Gu Y, Li M, Wang T, Liang Y, Zhong Z, Wang X, et al. Lactation-related microRNA expression profiles of porcine breast milk exosomes. PLoS ONE (2012) 7:e43691. doi: 10.1371/journal.pone.0043691

14. Modepalli V, Kumar A, Hinds LA, Sharp JA, Nicholas KR, Lefevre C. Differential temporal expression of milk miRNA during the lactation cycle of the marsupial tammar wallaby (Macropus eugenii). BMC Genomics (2014) 15:1012. doi: 10.1186/1471-2164-15-1012

15. Sedykh SE, Purvinish LV, Monogarov AS, Burkova EE, Grigor'eva AE, Bulgakov DV, et al. Purified horse milk exosomes contain an 
unpredictable small number of major proteins. Biochim Open (2017) 4:61-72. doi: 10.1016/j.biopen.2017.02.004

16. Yassin AM, Abdel Hamid MI, Farid OA, Amer H, Warda M. Dromedary milk exosomes as mammary transcriptome nano-vehicle: their isolation, vesicular and phospholipidomic characterizations. J Adv Res. (2016) 7:749-56. doi: 10.1016/j.jare.2015.10.003

17. Hock A, Miyake H, Li B, Lee C, Ermini L, Koike Y, et al. Breast milk-derived exosomes promote intestinal epithelial cell growth. J Pediatr Surg. (2017) 52:755-59. doi: 10.1016/j.jpedsurg.2017.01.032

18. Ma J, Wang C, Long K, Zhang H, Zhang J, Jin L, et al. Exosomal microRNAs in giant panda (Ailuropoda melanoleuca) breast milk: potential maternal regulators for the development of newborn cubs. Sci Rep. (2017) 7:3507. doi: 10.1038/s41598-017-03707-8

19. Liao Y, Du X, Li J, Lonnerdal B. Human milk exosomes and their microRNAs survive digestion in vitro and are taken up by human intestinal cells. Mol Nutr Food Res. (2017) 61:1700082. doi: 10.1002/mnfr.201700082

20. Simpson MR, Brede G, Johansen J, Johnsen R, Storro O, Saetrom P, et al. Human breast milk miRNA, maternal probiotic supplementation and atopic dermatitis in offspring. PLoS ONE (2015) 10:e0143496. doi: 10.1371/journal.pone.0143496

21. Izumi H, Tsuda M, Sato Y, Kosaka N, Ochiya T, Iwamoto H, et al. Bovine milk exosomes contain microRNA and mRNA and are taken up by human macrophages. J Dairy Sci. (2015) 98:2920-33. doi: 10.3168/jds.2014-9076

22. Chen T, Xi QY, Ye RS, Cheng X, Qi QE, Wang SB, et al. Exploration of microRNAs in porcine milk exosomes. BMC Genomics (2014) 15:100. doi: 10.1186/1471-2164-15-100

23. Lotvall J, Hill AF, Hochberg F, Buzas EI, Di Vizio D, Gardiner C, et al. Minimal experimental requirements for definition of extracellular vesicles and their functions: a position statement from the international society for extracellular vesicles. J Extracell Vesicles (2014) 3:10.3402/jev.v3.26913. doi: 10.3402/jev.v3.26913

24. Vlachos IS, Paraskevopoulou MD, Karagkouni D, Georgakilas G, Vergoulis T, Kanellos I, et al. DIANA-TarBase v7.0: indexing more than half a million experimentally supported miRNA:mRNA interactions. Nucleic Acids Res. (2015) 43:153-9. doi: 10.1093/nar/gku1215

25. Agarwal V, Bell GW, Nam JW, Bartel DP. Predicting effective microRNA target sites in mammalian mRNAs. Elife (2015) 4:e05005. doi: 10.7554/eLife.05005

26. Pathan M, Keerthikumar S, Chisanga D, Alessandro R, Ang CS, Askenase $\mathrm{P}$, et al. A novel community driven software for functional enrichment analysis of extracellular vesicles data. J Extracell Vesicles (2017) 6:1321455. doi: 10.1080/20013078.2017.1321455

27. Chen T, Xie MY, Sun JJ, Ye RS, Cheng X, Sun RP, et al. Porcine milk-derived exosomes promote proliferation of intestinal epithelial cells. Sci Rep. (2016) 6:33862. doi: 10.1038/srep33862

28. Benmoussa A, Lee CH, Laffont B, Savard P, Laugier J, Boilard E, et al. Commercial dairy cow milk microRNAs resist digestion under simulated gastrointestinal tract conditions. J Nutr. (2016) 146:2206-15. doi: 10.3945/jn.116.237651
29. Wolf T, Baier SR, Zempleni J. The intestinal transport of bovine milk exosomes is mediated by endocytosis in human colon carcinoma caco2 cells and rat small intestinal IEC-6 cells. J Nutr. (2015) 145:2201-6. doi: 10.3945/jn.115.218586

30. Golan-Gerstl R, Elbaum Shiff Y, Moshayoff V, Schecter D, Leshkowitz D, Reif S. Characterization and biological function of milk-derived miRNAs. $\mathrm{Mol}$ Nutr Food Res. (2017) 61:1700009. doi: 10.1002/mnfr.201700009

31. Kusuma RJ, Manca S, Friemel T, Sukreet S, Nguyen C, Zempleni J. Human vascular endothelial cells transport foreign exosomes from cow's milk by endocytosis. Am J Physiol Cell Physiol. (2016) 310:C800-7. doi: 10.1152/ajpcell.00169.2015

32. Lasser C, Alikhani VS, Ekstrom K, Eldh M, Paredes PT, Bossios A, et al. Human saliva, plasma and breast milk exosomes contain RNA: uptake by macrophages. J Transl Med. (2011) 9:9. doi: 10.1186/1479-5876-9-9

33. Patel V, Carrion K, Hollands A, Hinton A, Gallegos T, Dyo J, Sasik R, et al. The stretch responsive microRNA miR-148a-3p is a novel repressor of IKBKB, NF-kappaB signaling, and inflammatory gene expression in human aortic valve cells. FASEB J. (2015) 29:1859-68. doi: 10.1096/fj.14-2 57808

34. Liu X, Zhan Z, Xu L, Ma F, Li D, Guo Z, et al. MicroRNA148/152 impair innate response and antigen presentation of TLR-triggered dendritic cells by targeting CaMKIIalpha. J Immunol. (2010) 185:7244-51. doi: 10.4049/jimmunol.1001573

35. Iliopoulos D, Hirsch HA, Struhl K. An epigenetic switch involving NF-kappaB, Lin28, let-7 MicroRNA, and IL6 links inflammation to cell transformation. Cell (2009) 139:693-706. doi: 10.1016/j.cell.2009.10.014

36. Van Deun J, Mestdagh P, Sormunen R, Cocquyt V, Vermaelen K, Vandesompele J, et al. The impact of disparate isolation methods for extracellular vesicles on downstream RNA profiling. J Extracell Vesicles (2014) 3:10.3402/jev.v3.24858. doi: 10.3402/jev.v3.24858

37. EV-TRACK Consortium, Van Deun J, Mestdagh P, Agostinis P, Akay $\mathrm{O}$, Anand S, et al. EV-TRACK: transparent reporting and centralizing knowledge in extracellular vesicle research. Nat Methods (2017) 14:228-32. doi: $10.1038 /$ nmeth.4185

Conflict of Interest Statement: The authors declare that the research was conducted in the absence of any commercial or financial relationships that could be construed as a potential conflict of interest.

The reviewer PC declared a past co-authorship with several of the authors EH \& MW to the handling Editor.

Copyright (C) 2018 Herwijnen, Driedonks, Snoek, Kroon, Kleinjan, Jorritsma, Pieterse, Hoen and Wauben. This is an open-access article distributed under the terms of the Creative Commons Attribution License (CC BY). The use, distribution or reproduction in other forums is permitted, provided the original author(s) and the copyright owner(s) are credited and that the original publication in this journal is cited, in accordance with accepted academic practice. No use, distribution or reproduction is permitted which does not comply with these terms. 